Techniques \& Culture

Revue semestrielle d'anthropologie des techniques

37 | 2001

L'araire en Himalaya

\title{
Un araire du toit du monde
}

A swing plough (ard) of the top of the world

¿Un arado en el techo del mundo?

\section{Valérie Labbal}

\section{(2) OpenEdition}

\section{Journals}

Édition électronique

URL : https://journals.openedition.org/tc/261

DOI : $10.4000 /$ tc. 261

ISSN : 1952-420X

Éditeur

Éditions de l'EHESS

Édition imprimée

Date de publication : 1 juillet 2001

Pagination : 109-124

ISSN : 0248-6016

Référence électronique

Valérie Labbal, « Un araire du toit du monde », Techniques \& Culture [En ligne], 37 | 2001, mis en ligne le 20 octobre 2005, consulté le 29 septembre 2022. URL : http://journals.openedition.org/tc/261 ; DOI : https://doi.org/10.4000/tc.261

Ce document a été généré automatiquement le 29 septembre 2022.

Tous droits réservés 


\title{
Un araire du toit du monde
}

\author{
A swing plough (ard) of the top of the world \\ ¿Un arado en el techo del mundo?
}

Valérie Labbal

Je remercie P. Dollfus pour sa lecture critique de cet article et ses suggestions concernant l'étymologie des termes ladakhis, ainsi que J. Smadja qui a rendu possible le séjour au Ladakh au cours duquel une partie des informations contenues dans cet article ont été recueillies.

1 Le Ladakh (lat. $\mathrm{N}: 32-35^{\circ}$, long. $\mathrm{E}: 75-80^{\circ}$ ) est une région de hautes vallées qui se découpent au nord de la chaîne du Grand Himalaya et à l'extrémité occidentale du vaste plateau tibétain. Cette situation exceptionnelle, protégée par les reliefs, est la cause d'une forte aridité qui, en dehors des rives des cours d'eau, détermine le développement d'une végétation à caractère steppique. La population humaine, répartie dans de petites oasis le long des torrents et des rivières, pratique un mode de production agropastoral à base de cultures céréalières irriguées et d'élevage de bovidés. Le travail agricole est aujourd'hui encore réalisé à l'aide d'un faible nombre d'outils manuels et d'un seul instrument attelé, l'araire.

2 Situé à un point de rencontre des anciennes routes commerciales venues d'Inde, d'Asie Centrale et du Tibet, le Ladakh est une région au peuplement complexe mais qui, des points de vue culturel et linguistique, appartient au grand ensemble tibétain. Autrefois organisé en royaume indépendant ( $\mathrm{du} \mathrm{X}^{\mathrm{e}}$ au XIX ${ }^{\mathrm{e}}$ siècles), il fait aujourd'hui partie de l'État indien de Jammu-et-Cachemire. Il est divisé en deux districts, celui de Kargil qui borde le Cachemire et celui de Leh qui englobe la haute vallée de l'Indus. Nous proposons de décrire la structure, les conditions de fabrication, l'attelage, les fonctions et les techniques d'utilisation de l'araire en usage dans ce dernier district ${ }^{2}$. Cette nouvelle étude de cas permettra d'étendre le vaste champ de recherche sur les araires à des régions moins connues, caractérisées par leurs conditions extrêmes de milieu. 


\section{La structure de l'araire}

L'araire ladakhi appartient au type manche-sep de la classification établie par Haudricourt et Brunhes-Delamarre (1955) (fig. 1). Une unique pièce de bois courbée fait office à la fois de manche, c'est-à-dire d'appareil de gouverne, et de sep qui est l'élément supportant le soc. Cette pièce, appelée manche-sep, est percée d'orifices dans lesquels s'emboîtent les différentes parties de l'araire qui sont ensuite calées par des coins de bois. Cet araire étant destiné à être tiré par deux animaux, le manche-sep est traversé par un long timon de $250 \mathrm{~cm}$ en moyenne. Le soc est l'unique pièce de fer. Il est symétrique, de forme rectangulaire et légèrement convexe. Par son extrémité en forme de douille ouverte, il s'emmanche sur une tige de bois, fixée dans le sep. La partie du sep qui, au travail, glisse dans le fond de la raie est élargie par deux ailes constituées de simples planchettes de bois. L'araire est guidé à l'aide d'une poignée fixée perpendiculairement à l'extrémité du manche.

4 Le joug est une poutre de bois de coupe circulaire, d'environ $150 \mathrm{~cm}$ de long. Il repose à l'avant du garrot des deux animaux de trait auxquels deux colliers de corde permettent de le lier. Les parties du joug en contact avec les animaux sont généralement recouvertes de tissu.

5 Une boucle de corde constitue l'attache souple entre le timon et le joug. Elle est enroulée autour d'une pièce de bois fixée au centre du joug et d'une courte barre de bois qui traverse l'extrémité du timon. Les diverses manières de nouer cette boucle permettent d'ajuster la longueur de l'attache aux conditions d'attelage et de labour.

Chaque élément constitutif de notre araire est précisément désigné par un terme ladakhi que connaissent la plupart des hommes, familiarisés très tôt avec le maniement des outils agricoles et leur réparation. Les tableaux des figures 1 et 2 regroupent l'ensemble de ces termes sous leurs formes phonétique et translittérée ${ }^{3}$. Tout comme le tibétain dont il dérive, le ladakhi est une langue monosyllabique. Ce qui signifie qu'en règle générale, chaque mot composé de plus d'une syllabe peut être divisé en unités monosyllabiques possédant chacune un sens propre. La plupart des termes de notre nomenclature sont ainsi composés de deux syllabes qui possèdent un sens dans d'autres domaines que celui de la technique considérée : la "flèche de l'araire», le "fer de l'araire ", le «bois de la nuque ». Le seul terme spécifique de notre nomenclature est le terme gshol. Il désigne à la fois l'ensemble de l'instrument et le manche-sep, ce qui dénote l'importance de cette pièce qui est la plus caractéristique de notre araire.

Cet araire, que nous pouvons qualifier plus finement d'araire manche-sep, sans étançon ni genou, à timon long et à joug double de garrot, conserve la même allure dans tout le district de Leh. Ses proportions obéissent à une règle connue de tous les villageois et résumée par un dicton populaire : « $5,7,12$, celui qui ne connaît pas ces mesures n'est pas un bon chef de maison ${ }^{4}$ ». Ces trois chiffres correspondent respectivement aux mesures, traditionnellement évaluées avec la main et de ce fait non standardisées, du manche, du joug et du timon. Ils procurent un ordre de grandeur de ces trois pièces qui toutefois, dans la réalité, n'est pas rigoureusement respecté. En effet, les mesures d'araire effectuées montrent un manche plus court et de longueur variable (entre 70 et $95 \mathrm{~cm}$ ). La difficulté à se procurer une pièce de bois courbée de grande taille semble être à l'origine de ces écarts à la tradition. 
8 Au contraire de l'araire, le joug présente quelques variations de forme. Il en existe deux types (fig. 2) dont les aires de répartition coïncident avec une division locale qui distingue le Bas-Ladakh (Sham) du Haut-Ladakh (Stod). Ce découpage en deux contrées correspond à une réalité physique tout autant que culturelle ${ }^{5}$. Dans les villages du BasLadakh, les animaux de labour sont maintenus sous le joug par des baguettes de bois. Généralement, deux branches droites de tamaris (Myricaria), arbuste commun le long des cours d'eau, font l'affaire. D'autre part, la pièce de bois fixée au centre du joug a une forme évidée dans la région du Bas-Ladakh, qui diffère de la forme pleine de celle de la région du Haut-Ladakh.

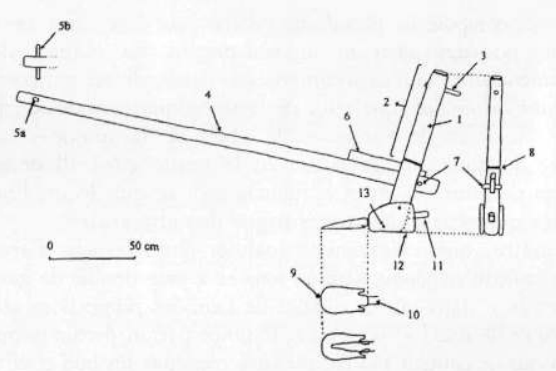

\begin{tabular}{|c|c|c|c|c|}
\hline $\mathbb{N}^{\circ}$ & $\begin{array}{l}\text { Terme ladakhi } \\
\text { phonétique }\end{array}$ & $\begin{array}{c}\text { Écriture selon la } \\
\text { translitération de Wvlie }\end{array}$ & Traduction littérale & Elément de l'araire \\
\hline 1 & shol & gstol & araire ou manche-sep & manche-sep \\
\hline 2 & sholgo & gabol-mgo & $\begin{array}{c}\text { araire-tête } \\
\text { (tête del'araire) }\end{array}$ & $\begin{array}{l}\text { partie supérieure } \\
\text { du manche-sep }\end{array}$ \\
\hline 3 & lakzoung & lag-bzung & main-saisir (poignée) & poignée \\
\hline 4 & sholda & gabol-mda' & $\begin{array}{c}\text { araire-fleche } \\
\text { (flèche de l'araire) }\end{array}$ & timon \\
\hline $5 \mathrm{a}$ & mik & mig & ceil & ceil percé à l'extrémité du timor \\
\hline 50 & mikshing & mig-shing & ceil-bois (bois de l'oeil) & $\begin{array}{l}\text { barre de bois à l'extrémité du } \\
\text { timon }\end{array}$ \\
\hline 6 & $\begin{array}{l}\text { konki ou } \\
\text { kong tsarks }\end{array}$ & $\begin{array}{c}\text { gong-kyi } \\
\text { ou } \\
\text { gong-brseegs }\end{array}$ & $\begin{array}{l}\text { au dessus-celui (celui [le bois] } \\
\text { d'au dessus) ou au dessus- } \\
\text { empilé (empilé au dessus) }\end{array}$ & $\begin{array}{l}\text { cale de bois qui maintient } \\
\text { le timon dans le sep }\end{array}$ \\
\hline 7 & $\begin{array}{c}\text { tsaspour ou } \\
\text { nakzer }\end{array}$ & $\begin{array}{l}\text { 11.phur ou } \\
\text { nas-gzer }\end{array}$ & $\begin{array}{c}\text { ?-cheville ou } \\
\text { noir-clou (clou noir) }\end{array}$ & $\begin{array}{l}\text { cheville de bois } \\
\text { al'arrière du timon }\end{array}$ \\
\hline 8 & shan & shan & cerceau & cerceau de métal \\
\hline 9 & shol tcharks & gshol-lcags & araire-fer (fer de l'araire) & soc \\
\hline 10 & tong tchoung & thong ${ }^{2}$-cbung & sep-petit (petit sep) & manche du soc \\
\hline 11 & djouki & mjug -kyi & $\begin{array}{c}\text { derrière-celui } \\
\text { (celui lle boiss de derrierre) }\end{array}$ & $\begin{array}{l}\text { cale de bois qui maintient le } \\
\text { soc dans le sep }\end{array}$ \\
\hline 12 & damlan & gram:33 & joue? & aile du sep \\
\hline 13 & damzèr & gram-gzer & joue-clou (clou de la joue) & clous des ailes \\
\hline
\end{tabular}

Figure 1. L'araire du Lakakh

91 Un point d'interrogation occupe la place des syllabes dont l'étymologie et conséquemment le sens n'ont pas été clairement établis.

$10{ }^{2}$ Thong est l'un des termes tibétains qui désignent l'araire (Jäschke 1881 ; Das 1902). Au Ladakh, ce terme est rarement utilisé dans le langage courant. Cependant, les dictionnaires ladakhis le définissent ainsi : «partie de l'araire dans laquelle le soc est placé » (Hamid 1998). Cette définition correspond au sep.

$11{ }^{3}$ L'érymologie de la syllabe « lan » demeure incertaine. Nous proposons : glan, « pièce rapportée (rajoutée) ». 

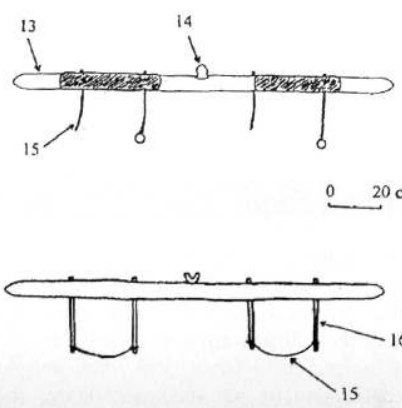

\begin{tabular}{|c|c|c|c|c|}
\hline $\mathrm{N}^{\circ}$ & $\begin{array}{l}\text { Terme ladakhi } \\
\text { phonétique }\end{array}$ & $\begin{array}{c}\text { Écriture selon la } \\
\text { translitération de } \\
\text { Wylie }\end{array}$ & Traduction littérale & Élément de l'araire \\
\hline 13 & nyashing & gma'shing & $\begin{array}{c}\text { nuque-bois } \\
\text { (bois de la nuque) }\end{array}$ & joug \\
\hline 14 & $\begin{array}{c}\text { spangpo ou } \\
\text { barmi }\end{array}$ & $\begin{array}{c}\text { dpang-po ou } \\
\text { bar-mi }\end{array}$ & $\begin{array}{l}\text { témoin ou } \\
\text { médiateur }\end{array}$ & $\begin{array}{c}\text { pièce de bois au centre du } \\
\text { joug }\end{array}$ \\
\hline 15 & pangtak & brang-tbag & $\begin{array}{c}\text { poitrine-corde } \\
\text { (corde de poitrine) }\end{array}$ & collier \\
\hline 16 & nyapour & gmya'pbur & cou-cheville de bois & barre de bois sous le joug \\
\hline 17 & kabtsé & khab-rtse & $\begin{array}{c}\text { aiguille-pointe } \\
\text { (pointe d'aiguille) }\end{array}$ & $\begin{array}{l}\text { soudure à l'extrémité du } \\
\qquad S O C\end{array}$ \\
\hline photo & $\begin{array}{l}\text { tchoutak } \\
\text { ou } \\
\text { dangpa }\end{array}$ & $\begin{array}{l}\text { goudthag } \\
\text { ou } \\
\text { ?.pa }\end{array}$ & $\begin{array}{c}\text { tortiller-corde } \\
\text { (corde * enroulée *) } \\
\text { ou } \\
\text { (courroie) } 1^{1}\end{array}$ & $\begin{array}{c}\text { attache } \\
\text { entre le timon et le joug }\end{array}$ \\
\hline photo & lang tchark & glang-icag & bøeuf-baguette & baguette \\
\hline
\end{tabular}

Figure 2. Les deux types de joug du Lakakh

${ }^{1}$ Le terme « dangpa ", dont l'étymologie nous est inconnue, désigne dans le Bas-Ladakh les courroies des métiers à tisser (information de P. Dollfus).

Ces différences structurelles s'accompagnent de quelques variations mineures dans la dénomination des pièces annexes de l'araire. C'est ainsi que dans la région du BasLadakh, la corde de liaison entre le joug et le timon est appelée « dangpa » (et non pas «tchoutak» comme dans le Haut-Ladakh) et la cale qui maintient le timon dans le sep est appelée « kong tsarks » (et non pas « konki »).

\section{La fabrication}

La fabrication d'un araire fait intervenir les techniques du bois et du fer qui, au Ladakh, étaient autrefois réservées à des groupes particuliers, endo-games: les musicienscharpentiers et les forgerons. Faisant l'objet d'interdits, notamment alimentaires et sexuels, et exerçant leur activité de manière héréditaire, ces derniers sont situés au plus bas de l'échelle sociale. Ils ne vivent cependant pas dans des villages spécifiques, mais se répartissent en marge de chaque oasis où ils se consacrent au service d'une communauté. Exempts des taxes et des charges qui incombent aux autres maisons de la communauté villageoise qui les abrite, ils reçoivent pour leur travail une part de la récolte de chaque maison.

Le travail du bois était autrefois réalisé par le groupe des musiciens (mon). Aujourd'hui, les membres de ce groupe se consacrent à l'accompagnement musical des divers événements sociaux et religieux qui animent la vie villageoise. L'activité du bois (construction d'araire, menuiserie) est exercée sans déshonneur par des villageois ordinaires qui, pour toute particularité, ont des aptitudes et du goût pour cette activité. Ils sont rémunérés au tarif en vigueur pour les travailleurs spécialisés ${ }^{6}$. Le bois utilisé 
dans la fabrication de l'araire provient pour l'essentiel des plantations privées de saules et de peupliers qui, dans chaque oasis, bordent les rives des cours d'eau et l'enceinte des maisons. Le saule est systématiquement préféré pour le timon. C'est, expliquent les villageois, un bois plus lourd mais plus solide que le peuplier. En ce qui concerne le sep et le joug, la nature du bois est très variable. Ce peut être du saule, du peuplier, et plus rarement du bouleau. Les petites pièces sont fabriquées avec du bois tout-venant $y$ compris le bois d'arbres fruitiers ${ }^{7}$. Les différents séjours sur place n'ont pas permis l'observation de la fabrication d'un araire. C'est en effet un outil qui dure, et la plupart des villageois assurent avoir toujours employé le même araire depuis qu'ils sont en âge de labourer. Cependant, après de plus amples discussions, beaucoup reconnaissent avoir changé au fil des années la majorité des pièces. Chaque villageois se charge luimême des réparations. Au cours des labours, la pièce qui rompt le plus fréquemment est la tige de bois sur laquelle s'emmanche le soc; elle est immédiatement remplacée par une nouvelle, taillée à l'herminette.

Le travail des métaux est aujourd'hui encore l'apanage du groupe des forgerons appelés littéralement « artisans du fer " («tcharks zopa », lcags-bzo-pa) ou plus communément, mais avec une connotation péjorative, "gara" (mgar-ba). Chaque village abrite au moins une maison de forgerons qui fabriquent, entretiennent, réparent l'outillage agricole et les ustensiles domestiques. En voici une illustration prise sur le vif.

Aujourd'hui 21 mars, Namgyal le forgeron se consacre au travail de réparation et de fabrication des outils agricoles de la maison Yokma. Trois membres de cette maison sont venus. Ils ont apporté le fer, le charbon de bois nécessaire à l'alimentation du feu de la forge et de quoi nourrir l'ensemble des personnes présentes tout au long de la journée de travail. Namgyal, de son côté, apporte son savoir-faire. Le foyer est installé à l'extérieur, dans un endroit dénudé, à proximité de sa maison. La fabrication d'un soc est en route ; elle se fait par martelage à chaud. Tout d'abord, Namgyal met la partie de la pièce en fer qui doit être travaillée à chauffer au rouge dans les braises ardentes. Assis près du foyer, il sort ensuite la pièce incandescente et la maintient à l'aide d'une pince sur une grosse pierre qui lui sert d'enclume. Puis il se contente d'indiquer de la pointe d'une tige de fer la partie à marteler. De leur côté, les villageois se relaient pour marteler la pièce avec la masse et actionner le soufflet en peau de chèvre [Les périodes de chauffage de la pièce durent de 1 à $2 \mathrm{mn}$; les périodes de martelage durent de 20 à $30 \mathrm{~S}$ pour une vingtaine de coups en moyenne]. La pièce est remise à chauffer dès qu'elle n'est plus rouge. La pièce ayant pris la forme rectangulaire désirée, Namgyal y pratique ensuite deux entailles qui partent des deux angles d'un même petit côté (fig. 3). Pour cela, il utilise un ciseau qu'il maintient à l'aide d'une deuxième pince pendant que l'un des villageois frappe ce même ciseau avec un marteau. Les deux entailles délimitent le soc proprement dit de la douille qui sera ensuite façonnée autour d'une barre de fer. Le soc, terminé au bout d'une heure et quart de travail, est pour finir immergé dans l'eau. Le travail de Namgyal se terminera par l'affûtage de nombreuses pioches et herminettes.

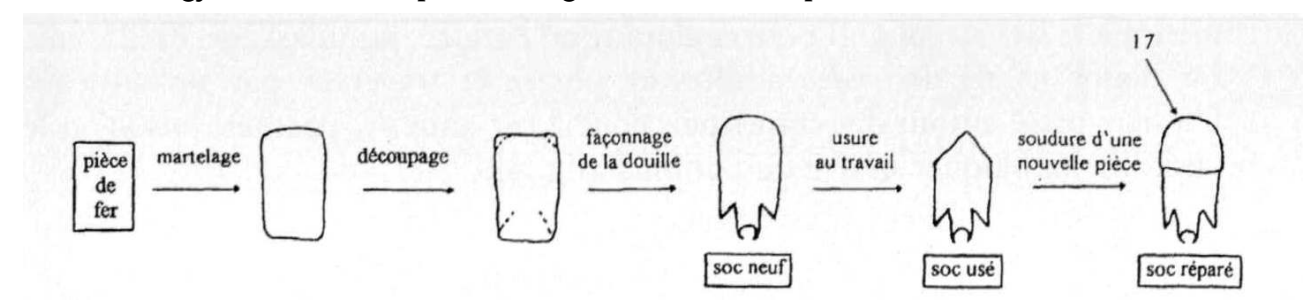

Figure 3. Fabrication et réparation d'un soc

17 Au fil des jours, le forgeron répète les mêmes gestes à l'aide des mêmes outils et fabrique des socs qui, dans une oasis donnée, sont tous de forme identique. Leur durée 
de vie dépend ensuite de l'intensité de leur utilisation (surface à labourer, nombre de labours par saison) et de la nature du sol (les sols sableux et pierreux usent les outils plus rapidement). En moyenne, ils sont changés ou réparés tous les deux à trois ans. Les socs usés sont parfois réparés par soudure d'une nouvelle pièce de fer à l'extrémité du soc. Cette solution, autrefois préférée du fait des difficultés à se procurer du fer, est aujourd'hui souvent écartée. Les villageois jugent en effet que les socs réparés sont plus lourds et moins équilibrés, ce qui rend l'araire plus difficile à manier.

\section{L'attelage}

18 L'animal de labour, le $m d z o$, est issu du croisement d'un yak (Bos grunniens) et d'une vache (Bos taurus). Plus docile que le yak, il est également mieux adapté aux altitudes du Ladakh $^{8}$ Peu de yaks sont visibles dans les oasis du district de Leh. Chaque village en possède un ou deux qui servent exclusivement de géniteurs. En revanche, la plupart des maisons possèdent un ou deux $m d z o$. Différentes formes traditionnelles de coopération entre maisons existent, qui permettent à chacune de disposer d'un couple de mdzo à l'époque des semailles. La première forme, appelée «langde » (glang-'dres, «mélanger les bœufs »), associe deux maisons qui mettent en commun leur main-d'œuvre familiale et leurs animaux de labour pour la durée de la saison agricole. La deuxième forme, appelée « dzopès » ( $m d z o-p h y e s$, « diviser les $m d z o »)$, associe une maison sans animal de labour avec une autre qui en possède deux. Le prêt des bêtes pat cette dernière est alors compensé par le prêt de main-d'œuvre de la part de la première. Aujourd'hui, un nombre croissant de maisons préfère louer les services d'un villageois et de ses $m d z o$. Il leur en coûte $70 \mathrm{Rp}$ par jour pour le travail du laboureur et $200 \mathrm{Rp}$ pour la location des deux animaux (tarifs de 1996). Cette solution attire principalement les maisons caractérisées par une main-d'œuvre familiale réduite et des revenus monétaires réguliers.

De la naissance à l'âge de 5 ans, le jeune $m d z o$ appelé « dzoubi » ('dzu-bi) ne participe pas au labour. Ce n'est qu'à partir de 5 ans que l'on commence à le lier au joug. Il pourra alors tirer l'araire jusqu'à l'âge de 25 ans. La cloison nasale des $m d z o$ adultes est percée et traversée par un anneau. Un lien, passé autour des cornes puis noué à cet anneau, permet, lorsqu'on le saisit, de lui bloquer la tête de l'animal (fig. 4). 


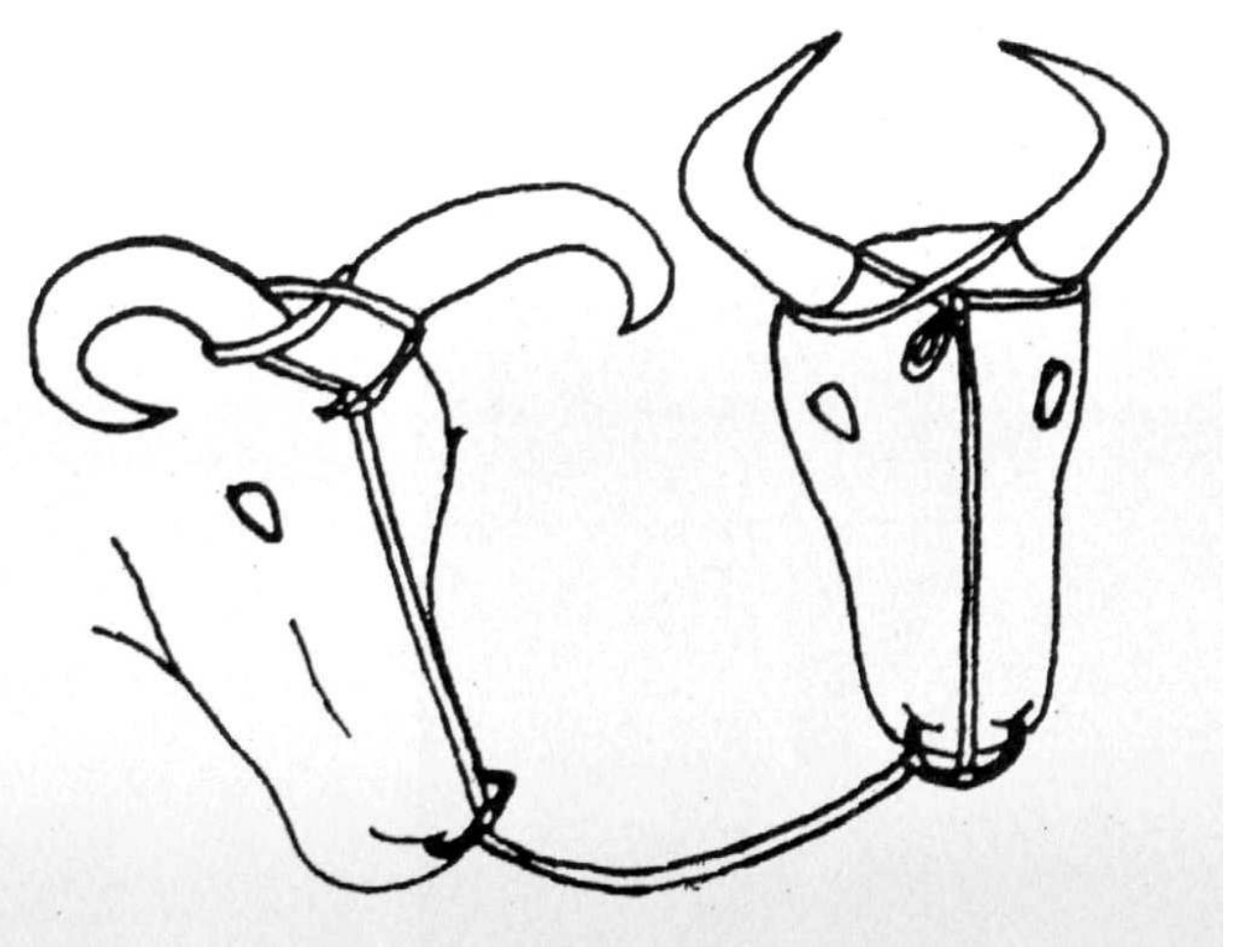

Figure 4. Paire de dzo prêts à être enjugués

Avant d'être enjugués, les deux mdzo de l'attelage sont attachés ensemble par un nouveau lien qui passe dans leurs anneaux de nez. Pendant qu'un homme les maintient, un autre se glisse entre eux et leur pose le joug sur le cou, à l'avant du garrot. Le joug est ensuite attaché au cou des deux animaux puis le timon est fixé sous le joug par une boucle de corde (fig. 5). Les jeunes qui n'ont jamais tiré l'araire sont entraînés avant le début de la saison agricole. Cette période d'exercice est appelée « l'entraînement du cou » («nyabjang », gnya'-sbyang). Le jeune mdzo est alors lié avec un congénère aguerri à un joug qui, lorsque c'est possible, est plus long que dans la pratique. Un long joug, outre le fait qu'il est plus difficile pour les bêtes de s'en dégager, permet de maintenir une distance plus grande entre les deux animaux qui, peu habitués l'un à l'autre, essayent de se battre. Le nom de "médiateur " porté par la pièce de bois au centre du joug prend alors tout son sens. Au cours de l'exercice, la présence d'un individu adulte est indispensable pour maintenir les $m d z o$. Dans le cas contraire, les mouvements désordonnés des deux animaux risquent d'entraîner la rupture du joug. 


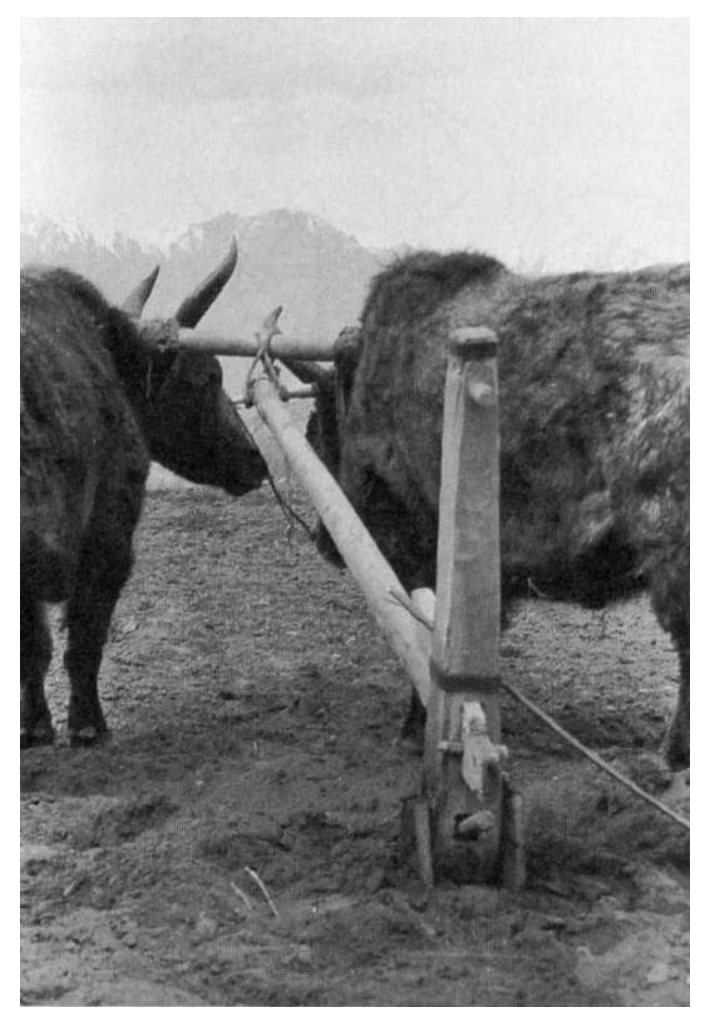

Figure 5. Système d'attache du timon au joug

\section{La place de l'araire dans l'itinéraire technique}

Le tracé ritualisé d'une première raie avant la saison des semailles est une cérémonie largement répandue dans toute l'Inde et la Chine. Au Ladakh, elle a pour nom "ouverture de la bouche de la terre" («sakapé», sa-kha-'bye). À une date faste, déterminée par l'astrologue de chaque village, la terre est symboliquement ouverte à l'araire. À cette occasion, tous les mdzo des villageois, brossés, généreusement nourris et les cornes ornées de morceaux de beurre, sont enjugués tour à tour. Leurs propriétaires espèrent ainsi attirer une fortune favorable sur les activités agricoles qui, dès lors, peuvent débuter.

L'araire intervient pour la première fois dans l'itinéraire technique ${ }^{9}$ au moment des semailles. Son rôle consiste à ameublir le sol et enfouir les semences. Ce type de labour associé au semis porte un nom spécifique : «ngo » $(\mathrm{rmo})$. C'est seulement quand elle est combinée à d'autres techniques que l'action de I'araire sur le sol devient véritablement efficace. La saison débute donc réellement par une irrigation qui prépare le sol au futur labour. Ensuite, au moment du semis, le labour à l'araire ouvre le sol et l'émiette. Les plus grosses mottes sont a lots brisées à la pioche ou au pied. Enfin, le travail au brisemotte (mince planche de bois fixée perpendiculairement à l'extrémité d'un long manche) achève l'émiettement du sol et aplanit la surface semée. L'irrigation préalable au labour, appelée « eau [du sol] gris» («skyatchou», skya-chu), est essentielle. À tel point qu'il est commun que les Ladakhis qui rencontrent des laboureurs à l'époque des semis les saluent en ces termes : "que l'humidité vous soit favorable ». En fonction de la texture du sol, l'intervalle de temps entre l'irrigation et le labour est plus ou moins long. Les plus mauvais champs doivent être labourés quelques jours après l'irrigation. 
En revanche, les propriétaires de bons champs, régulièrement fumés, peuvent attendre jusqu'à un mois avant de labourer. Une fois le champ labouré, semé et plané, l'araire permet en quelques minutes de tracer les rigoles d'irrigation qui compartimentent la parcelle. Les champs de céréales sont généralement labourés une deuxième fois à l'automne, après la récolte. Dans l'oasis de Sabu, ce labour n'est réalisé qu'en dessous de $3600 \mathrm{~m}$ d'altitude. Les villageois expliquent qu'il est un bon moyen de lutter contre une adventice à fortes racines (ram-pa, chiendent), peu envahissante à des altitudes supérieures. Ce labour d'automne, réalisé par un seul individu, est appelé «briser le champ» («jing lok», zhing-rlog ${ }^{10}$. Dans les oasis du fond de la vallée de l'Indus, caractérisées par un sol plus argileux, le labour d'automne est associé à une irrigation qui le précède en vue d'ameublir la terre.

Enfin, l'araire est utilisé pour la récolte des pommes de terre. Un labour à l'araire ouvre la terre. À l'aide d'une fourche de bois, deux ou trois travailleurs éjectent les pommes de terre hors de la raie fraîchement tracée; quelques autres les ramassent. Ce labour ne porte pas de nom spécifique; les Ladakhis désignent simplement l'ensemble de l'opération par l'expression "sortir les pommes de terre » ("alou bing», a-lu [terme hindi] 'byin).

\section{La technique de labourage}

La description précise d'actes techniques est bien plus difficile lorsque l'observation et l'enquête orale ne peuvent s'enrichir d'une expérimentation personnelle. Mais lorsque le sacré s'en mêle, le chercheur n'a plus le choix. Or, dans toute la région du Ladakh, la conduite de l'araire est exclusivement réservée aux hommes. Les Ladakhis considèrent que tout manquement à cette règle, en dérangeant l'ordre établi du monde, pourrait être générateur de troubles et de malheurs pour l'ensemble de la communauté. La tradition orale rapporte par ailleurs l'histoire d'une femme qui, devant le manque cruel de main-d'œuvre masculine dans sa maisonnée, aurait osé braver l'interdit. Elle fut immédiatement sanctionnée par l'interruption de toutes les relations sociales qui l'unissaient au reste des villageois.

La description qui suit résulte donc d'observations et d'enquêtes. Elle concerne le labour-semis. Un homme (thong-pa, «le laboureur») conduit l'araire d'une main. Il marche à côté de l'instrument, dans la partie déjà labourée (fig. 6). 


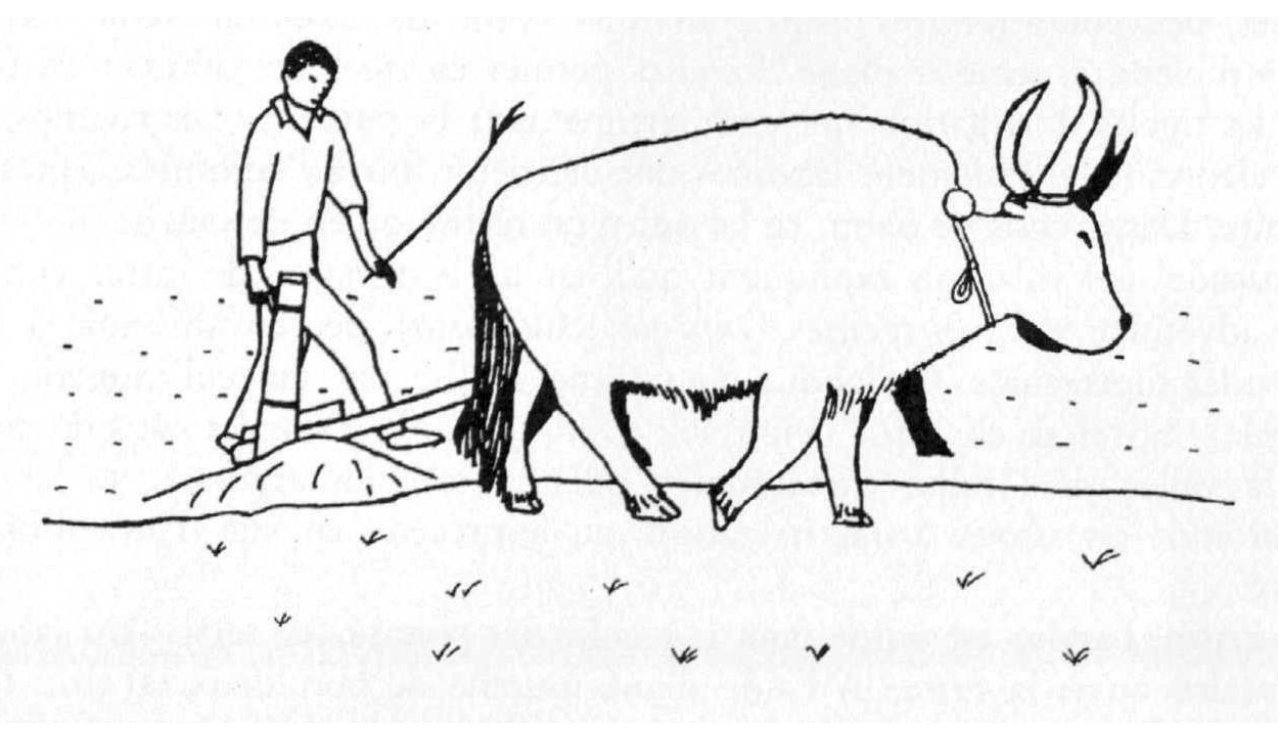

Figure 6. La position du laboureur

Pour ce faire, il conduit l'araire tantôt de la main droite, tantôt de la main gauche. De sa main libre, il brandit une branche de saule (« lang tchark », glang-lcag, «baguette pour le bœuf ») dont il use pour stimuler le couple de $m d z o$. Lorsque les bêtes sont peu dociles ou le laboureur novice, un individu (« dzotitkan », mdzo 'khrid-mkhan, « celui qui mène les $m d z o »)$ se place en tête de l'attelage. Il guide alors les animaux en tirant sur la corde qui relie leurs anneaux de nez. En arrière du laboureur, un deuxième individu ( «sa on pa », sa-bon-pa, «l'homme des graines ») suit et sème dans la raie (« rol la tab», rol-la btab, «jeter dans la raie »). Une à deux poignées de graines sont jetées en trois gestes de la main. Chaque raie (rol) est recouverte de terre par le tracé de la raie suivante. Les graines sont alors enfouies à $7 \mathrm{ou} 8 \mathrm{~cm}$ de profondeur. Au moment des semailles, le rôle de l'araire consiste donc en un ameublissement du sol (jusqu'à $15 \mathrm{~cm}$ environ), le tracé de raies et le recouvrement des semences. Un labour mal fait est un labour qui échoue à recouvrir les semences. Deux raies sont alors simultanément visibles après le passage de l'araire. Les jeunes laboureurs qui manquent d'expérience laissent fréquemment de courtes bandes de terre non travaillées entre deux raies. Ces bandes, désignées du même nom que les traces occasionnées par un coup de fouet (nya), leur valent les railleries de leurs aînés.

La position classique du laboureur, précédemment décrite, ne permet pas toujours de fournir une pression suffisant au maintien dans le sol des parties travaillantes de l'araire. C'est ainsi qu'au moment d'entamer la raie, le laboureur augmente parfois la pression exercée par sa main, de celle de son pied. Il place alors un pied sur la partie horizontale $\mathrm{du}$ sep, l'autre pied demeurant en l'air en arrière du corps. Cette position dure le temps de ficher le soc en terre, lorsque les animaux amorcent leur mouvement. Les bordures du champ, plus difficiles à travailler, nécessitent parfois une pression accrue. Le laboureur appuie alors de tout son poids sur l'araire, une main sur le manche, l'autre sur le timon (fig. 7). 


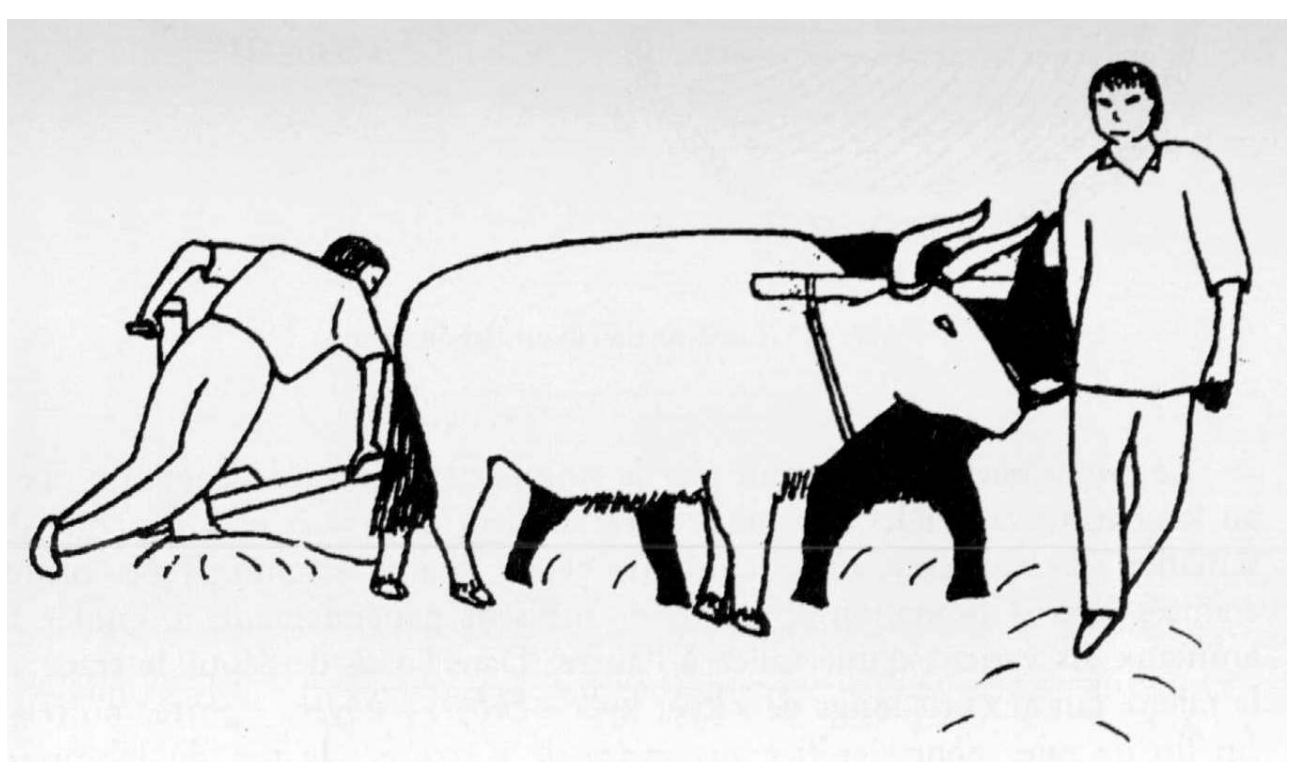

Figure 7. Tracé d'une raie en bordure de champ

La géométrie du labour montre que l'attelage effectue des va-et-vient selon des lignes courbes qui épousent les formes de la parcelle (fig. 8). Hormis dans les petits champs, les passages de l'araire ne sont pas effectués suivant la plus grande longueur. Un laboureur préfère en effet réaliser des raies au tracé plus court mais plus précis ${ }^{11}$. La longueur des raies dépend également de la qualité du sol. Un sol trop sec épuise hommes et bêtes qui effectueront par conséquent des raies plus courtes. En revanche, un sol bien préparé autorise des raies plus longues. C'est en ce sens que certains villageois interprètent le dicton «si le laboureur tombe, le champ portera une bonne récolte ». Dans un bon champ, le tracé de longues raies réalisées à une vitesse plus rapide et le caractère meuble du sol labouré augmentent en effet la probabilité de trébucher. Enfin, le dessin du labour épargne tout autour des champs une bande de terre nécessaire aux demi-tours de l'attelage.

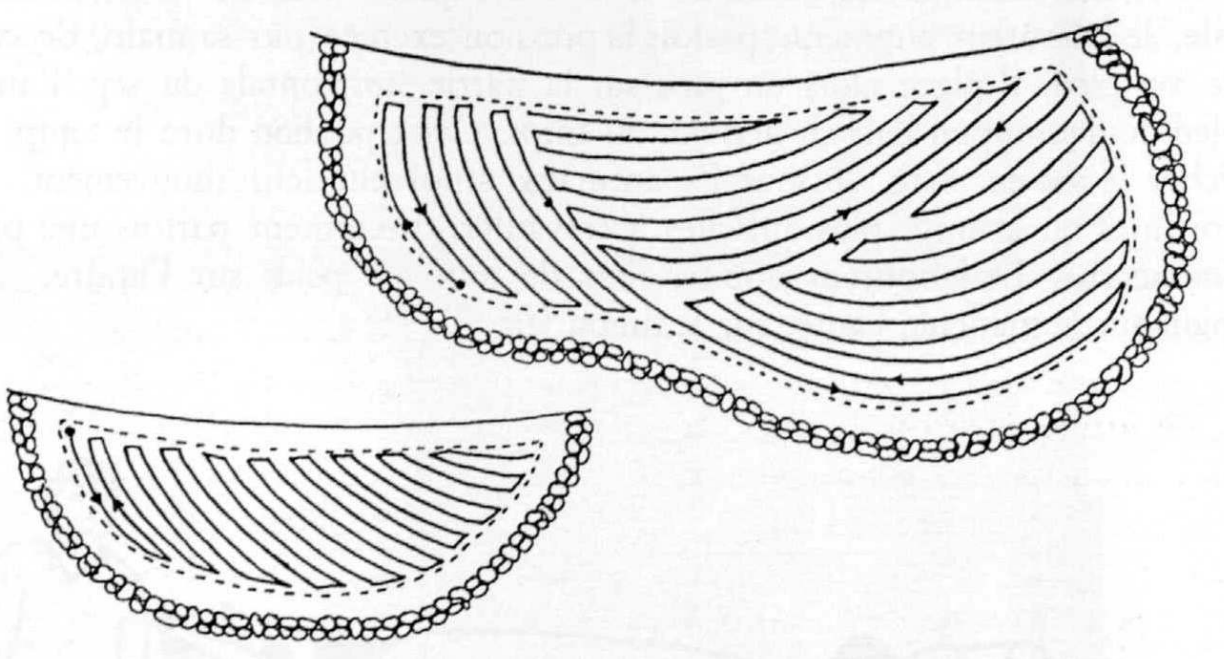

Figure 8. Géométrie du labour (labour-semis)

Le laboureur ne se contente pas de stimuler ses bêtes à la baguette. Tout au long du travail, il les encourage constamment de la voix et à l'époque des semailles, les vallées 
résonnent de cris et de chants sonores. Trois ordres, énoncés avec l'intonation appropriée, suffisent généralement à guider les animaux. Ils varient d'une vallée à l'autre. Dans l'oasis de Sabu, le tracé de la raie se fait aux cris longs de «kyèr kyèr " (khyer- khyer, «porte, porte »). En fin de raie, pour signifier aux mdzo de s'arrêter, le ton du laboureur s'infléchit vers le bas lorsqu'il prononce d'une voix grave «a ri ri » (expression dont le sens nous est inconnu). Enfin, le laboureur enjoint ses bêtes de tourner par les cris plus secs de "khor khor " ('khor-'khor, "tourne, tourne »). À ces trois ordres, s'ajoutent de multiples encouragements et parfois des chants par lesquels les $m d z o$, qualifiés de divers surnoms affectueux, sont remerciés de leurs efforts. Autrefois, des musiciens itinérants se déplaçaient d'une équipe de labour à l'autre pour entonner en l'honneur des $m d z o$ un long chant traditionnel. Ce chant célébrait la beauté des pâturages d'altitude qui attendent les $m d z o$ une fois les labours terminés.

La technique du labour-semis n'est pas homogène dans tout le district de Leh. Il arrive dans certains cas que le semeur précède l'attelage et jette les graines dans la raie tracée lors du précédent passage, tout en servant de guide aux bêtes. Cet arrangement est cependant peu apprécié, car le semeur ne peut ni voir, ni contrôler les mouvements de tête des animaux. D'autre part, le Bas-Ladakh se caractérise par la fréquence d'une autre technique : le semis à la volée, appelé « gyastor » (rgya-gtor, « jeter large »).

L'araire que nous venons de décrire convient bien à certaines exigences des milieux de montagne. Il est léger (un homme seul peut le porter sur l'épaule de terrasses en terrasses) et maniable (il est utilisé dans des champs de taille et de forme diverses). D'agencement simple, il est réparé très rapidement, souvent sur le lieu même du travail. D'un point de vue fonctionnel, il est intéressant de noter que cet araire est systématiquement l'instrument des semailles. Par ailleurs, l'itinéraire technique se caractérise par un faible nombre de passages de l'araire. L'efficacité de ce travail du sol peu intensif est cependant améliorée par des applications préalables d'eau.

Si l'on considère la classification de Haudricourt et Brunhes-Delamarre, le Ladakh se rattache au vaste domaine de répartition des araires manche-sep. L'araire ladakhi appartient plus précisément au type le plus caractéristique des araires manche-sep, celui dont le manche-sep est véritablement constitué d'une pièce unique. Ce type paraît largement répandu des oasis d'Asie Centrale au Tibet, dans des régions arides d'agriculture céréalière irriguée. L'araire ladakhi se singularise cependant par l'absence $\mathrm{du}$ " genou ", petite pièce de renfort reliant transversalement le timon au manche-sep. Le problème de l'introduction de l'araire au Ladakh se pose, d'autant plus qu'il est actuellement l'unique instrument aratoire employé dans la région et que, mises à part les plantations d'arbres récentes, le bois est une ressource rare au Ladakh. La tradition locale apporte peu d'informations à ce sujet; elle se réfère à la tradition tibétaine, qui attribue l'apparition de l'araire au règne légendaire de sPu-lde gung-rgyal ${ }^{12}$. 


\section{BIBLIOGRAPHIE}

Das, S.C.

1902, A Tibetan-English Dictionary with Sanskrit synonyms. Delhi : Book Faith India (réédition 1992).

Dollfus, Pascale

1989, Lieu de neige et de genévriers. Paris : Éditions du CNRS.

Hamid, A.

1998, Ladakhi-English-Urdu Dictionary. Leh : Melong Publications.

Haudricourt, André-Georges et Mariel Brunhes-Delamarre

1955, L’homme et la charrue à travers le monde. Lyon : La Manufacture (réédition 1986).

Jäschke, $\mathrm{H}$.

1881, A Tibetan-English Dictionary with special reference to the prevailing dialects. Delhi : Motilal

Banarsidass Publishers (réedition 1992).

Milleville, P.

1987, «Recherches sur les pratiques des agriculteurs », Les Cahiers de la Recherche Développement

$16: 3-21$.

Sebillote, M.

1978, « Itinéraires techniques et évolution de la pensée agronomiques », C.R. Académie agriculture

française 11 : 906-913.

\section{NOTES}

2. Des observations précises ont été menées dans l'oasis de Sabu, proche de la ville de Leh, dont les champs arrosés par un petit torrent s'étagent en terrasses de 3400 à $3900 \mathrm{~m}$ d'altitude. Ces observations ont été complétées par des enquêtes réalisées dans d'autres oasis du district de Leh.

3. Les formes translitérées, précisées en italique dans le texte et dans les tableaux, correspondent à l'orthographe tibétaine. La translittération implique donc que l'étymologie tibétaine de chaque terme ladakhi (si elle existe) soit connue, ce qui n'est pas toujours le cas. Dans le texte, la forme phonétique sera précisée lorsqu'elle diffère grandement de la forme translittérée.

4. La société ladakhie est organisée en «maisons ». An sens ethnologique du terme, la maison, détentrice de biens matériels et immatériels, d'un statut et d'un nom, est une véritable personne morale qui possède des droits et des devoirs au sein de la communauté à laquelle elle appartient. C'est au nom de la maison que le chef de maison ou un autre de ses membres participe aux affaires du village. Enfin, elle constitue l'unité de production agricole et détient l'ensemble des outils nécessaires à cette production, parmi lesquels l'araire. Sur l'organisation sociale au Lakakh, consulter Dollfus (1989).

5. Le lit de l'Indus, très encaissé entre ces deux contrées, oblige le voyageur à un détour par un plateau désertique qui redescend ensuite très fortement en direction du Bas-Ladakh. De légères variations dans la prononciation et le vocabulaire différencient le parler des populations installées de part et d'autre de ce passage.

6. Les villageois spécialisés dans le travail de la maçonnerie ou du bois étaient rémunérés 150 roupies indiennes (Rp) par jour en 1996 par ceux qui louaient leurs services. À la même époque, les ouvriers agricoles masculins étaient rémunérés $70 \mathrm{Rp}$ et leurs homologues féminines, $65 \mathrm{Rp}$. Tous les travailleurs ladakhis sont nourris le temps de leur engagement. 
7. Le bouleau se développe à l'état naturel dans certaines parties du Ladakh. Dans le district de Leh, il est cependant très difficile de s'en procurer. Quelques maisons possèdent des pommiers et des abricotiers plantés à des altitudes inférieures à $3500 \mathrm{~m}$ (l'un des araires observés avait des ailes en bois de pommier).

8. Le yak préfère des altitudes supérieures à $3500 \mathrm{~m}$.

9. L'itinéraire technique, équivalent agronomique de la chaîne opératoire des ethnologues, est défini comme une « combinaison logique et ordonnée de techniques qui permettent de contrôler le milieu et d'en tirer une production donnée » (Sébillote 1978 ; Milleville 1987).

10. L'étymologie généralement admise pour «jing lok» est zbing-log, « retourner le champ ». Cependant, zbing-rlog « briser le champ » nous semble mieux correspondre à la réalité.

11. En revanche, le labour d'automne et le labour à la récolte des pommes de terre exigent moins de précision. De longues raies sont alors effectuées.

12. La période historique au Tibet ne débute qu'à partir du VII ${ }^{\mathrm{e}}$ siècle de notre ère. Pour la période qui précède, les Chroniques du Tibet et du Ladakh énumèrent, après une série de sept rois « célestes ", une succession de rois mythiques (sPu-lde gung-rgyal est le second de cette série) et assignent au règne de chacun divers progrès matériels.

\section{RÉSUMÉS}

L'araire du Ladakh (Himalaya occidental indien) se rattache au vaste domaine de répartition des araires manche-sep. L'article en décrit la structure, la fabrication, ses fonctions et ses techniques d'utilisation. Il donne également la nomenclature associée à cet outil et celle du joug qui l'accompagne.

The Ladakhi (Indian Western Himalayas) swing plough (ard) belongs to the vast distribution zone of shaft-stock swing ploughs. The present paper describes the structure of the implement, its fabrication, its functions and the techniques of use. This article also gives the related nomenclature as well as that of the associated yoke.

El arado del Ladakh (Himalaya occidental índico) se relaciona con el amplio campo de distribución de los arados «mancera-dental ». El artículo describe su estructura, su fabricación, sus funciones y sus técnicas de utilización. También presenta la nomenclatura que respecta a este instrumento y la del yugo con que se utiliza.

INDEX

Mots-clés : agriculture, araire, Himalaya, Ladakh

\section{AUTEUR}

\section{VALÉRIE LABBAL}

Agronome, doctorante en anthropologie, Université de Provence 